\title{
Pion Decay Widths of $D$ mesons
}

\author{
K.O.E. Henriksson, ${ }^{*}$ T.A. Lähde ${ }^{\dagger}$ C.J. Nyfält ${ }^{\ddagger}$ and D.O. Riska ${ }^{\S}$ \\ Department of Physics \\ and \\ Helsinki Institute of Physics, POB 9, 00014 University of Helsinki, Finland
}

\begin{abstract}
The pionic decay rates of the excited $L=0,1 \mathrm{D}$ mesons are calculated with a Hamiltonian model within the framework of the covariant Blankenbecler-Sugar equation. The interaction between the light quark and charm antiquark is described by a linear scalar confining and a screened one-gluon exchange interaction. The decay widths of the $D^{*}$ mesons obtain a contribution from the exchange current that is associated with the linear scalar confining interaction. If this contribution is taken into account along with the single quark approximation, the calculated decay rates of the charged $D^{*}$ mesons are readily below the current empirical upper limits if the axial coupling constant of the light constituent quarks is taken to be $g_{A}^{q}=0.87$, but reach the empirical upper limits if $g_{A}^{q}=1$. With the conventional values for $g_{A}^{q}$, the calculated widths of the $D_{1}$ and $D_{2}^{*}$ mesons fall somewhat below the experimental lower limits, leaving room for other decay modes as well, such as $\pi \pi$ decay. The unrealistically large contribution from the axial charge operator to the calculated pion decay width of the $D_{1}$ meson is suppressed by taking into account the exchange charge effects that are associated with the scalar linear confining and vector one-gluon exchange interactions. The predicted values for the pionic widths of the hitherto undiscovered $L=1 D_{1}^{*}$ and $D_{0}^{*}$ mesons are found to be smaller than previous estimates.
\end{abstract}

\footnotetext{
*koehenri@pcu.helsinki.fi

†talahde@pcu.helsinki.fi

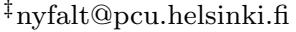

§riska@pcu.helsinki.fi
} 


\section{Introduction}

The pion decay rates of the excited charmed mesons - the $D$ mesons - may provide direct information on the strength of the pion coupling to light constituent quarks. As the charm quark in the $D$ mesons does not couple to pions, the decay mechanism is determined by the pion coupling to the light flavor constituent quark. A first assumption is that this coupling is independent of the interaction between the light quark (or antiquark) and the charmed antiquark (or quark). While this may be considered as a satisfactory approximation for the axial current part of the pion-quark coupling, it leads to large overestimates of the decay widths in the case of the axial charge term, a problem that may be cured by the two-quark mechanism that is associated with the confining interaction between the light and charmed quark in the $q \bar{c}(\bar{q} c)$ system.

Because of the large velocities of the confined quarks in $D$ mesons, and in view of the small mass of the light constituent quarks, the $D$ mesons have to be described as relativistic interacting two-particle systems. The simplest way to achieve such a description is to employ a covariant three dimensional reduction of the Bethe-Salpeter equation and a corresponding quasipotential representation of the interaction between the constituents. The hitherto considered quasipotential descriptions for the $D$ mesons are based on the Gross [1] and the Blankenbecler-Sugar equations [2, 3]. Both approaches have been shown to yield reasonable predictions for the $D$ meson spectrum with combinations of linear confining and one-gluon exchange interactions between the quark and antiquark [4, 5, 6].

This dynamical model, with static interactions in the $q \bar{Q}$ system applied in the solution of the Gross equation, has recently been used to calculate the pseudoscalar meson decay rates of the $D$ mesons [8]. A fair description of the decay rates of the $D^{*}$ mesons was achieved, under the assumption that the pions couple to the light constituent quarks by the standard (pseudovector) coupling. On the other hand, the ratio of the widths of the $D_{2}^{*}(2460)$ and the $D_{1}(2420)$ mesons was found to be $\sim 2.5$, while the empirical ratio is $\sim 1.3$. In view of the importance of determining the form and strength of the pion-quark coupling, an analogous calculation within the framework of the Blankenbecler-Sugar equation is performed here. There is no obvious reason for preferring one or the other quasipotential framework, besides that of calculational convenience. The Gross equation framework has the virtue of reducing to a Dirac equation for the light quark in the infinite mass limit of the heavy (charm) quark. The Blankenbecler-Sugar equation has an interpretational advantage in its formal similarity to the standard Schrödinger equation framework.

To calculate the pion decay rates of the excited $D$ mesons, the wavefunctions that have been obtained in ref. [6] by solving the Blankenbecler-Sugar equation in configuration space are employed in order to achieve a description of the states in the $D$ meson spectrum. These wavefunctions correspond to a model which describes the interaction in the $q \bar{Q}$ system as a scalar linear confining interaction combined with a screened relativistic one-gluon exchange interaction, which yields a spectrum that agrees with the empirically known part of the spectrum. The model leads to hyperfine splittings that agree well with recent NRQCD lattice calculations in the quenched approximation [9]. The rates for the decays of the form $D^{\prime} \rightarrow D \pi$ are then obtained by calculating the matrix elements of the pion creation operator between the excited and ground states using such wave functions. Because of the small mass of the light flavor quark, which couples to the pions, the non-local structure of the pseudoscalar coupling of the pion to the quark has to be treated in unapproximated form. The static (local) approximation to this vertex function is shown to imply an overestimate by about a factor 2 .

The present empirical information on the widths of the excited charm mesons remains very incomplete. Absolute values, with large uncertainty ranges, are known for the decay widths of the $D_{1}(2420)$ and $D_{2}^{*}(2460)$ mesons, but for the $D^{*}(2010)^{ \pm}$and the $D^{*}(2007)^{0}$ only upper limits are available at 
present [12]. The pion decays of the $D^{*}$ mesons to $D$ mesons are $P$-wave decays generated by the axial current operator. If the pion is assumed to be emitted by a single quark operator with the conventional value for the pion-quark coupling constant, the calculated widths for the decays $D^{*} \rightarrow D \pi$ fall well below the present empirical upper limits in the case of the charged $D^{*}$ mesons (The empirical upper limit on the width of the neutral $D^{*}$ meson is too large to be constraining). Upon addition of the contribution from the two-body axial exchange (pair) current that is associated with the linear scalar confining interaction, the calculated widths reach the empirical upper limits in case of the charged $D^{*}$ mesons, if the value of the axial coupling constant of the light constituent quarks is taken to be $g_{A}^{q}=1$.

The single quark mechanisms for pion production lead to a considerable overprediction of the $S$-wave pion decay widths of the $D_{1}$ mesons. This overestimate may be reduced by invoking the two-quark mechanism, which is naturally associated with the scalar confining and vector one-gluon exchange interactions. Consequently, the predicted widths of other $D$ meson states that decay by an $S$-wave mechanism are also suppressed by large factors. This effect was first hinted at in ref. [8]. In the case of the scalar confining interaction the simplest description of this mechanism is to view it as an effective increase of the constituent quark mass from $m_{q}$ to $m_{q}+c r$, where $c$ is the confining string tension. Since the quark mass appears in the denominator of the transition amplitude for $S$-wave pion decay, this increase of the constituent quark mass leads to a large reduction of the associated matrix element. An analogous suppression of $S$-wave pion decay modes was achieved in ref. [8] as a correction to the one-quark operator through coupling to negative energy states. This two-body mechanism is analogous to that, which is required for a realistic description of the M1 decays of charmonium and heavy light mesons [6, 7].

Once this two-quark mechanism is taken into account, the calculated pion decay widths of the $D_{1}(2420)$ and $D_{2}^{*}(2460)$ mesons fall somewhat below the empirical values if the axial coupling of the constituent quark is taken to be less than 1. If, on the other hand, the matrix element of the pion decay amplitude is evaluated in the non-relativistic approximation, the calculated decay widths exceed the empirical values, in agreement with the result of ref. [8]. In the case of the $D_{1}$ meson, the $S$-wave pion decay mode is found to contribute significantly, so that in the end the net ratio of the calculated widths of the $D_{2}^{*}$ and $D_{1}$ mesons is about 1.2 , which falls within the wide uncertainty range of the current experimental value 1.3. The underprediction of the pionic decay widths of the $L=1 \mathrm{charm}$ mesons is natural, as a substantial fraction of the total width is expected to be due to other decay modes, in particular $\pi \pi$ decay. The analogy with the corresponding decay modes of the $K_{2}^{*}(1430)$ strange meson suggests that the decay modes $D^{\prime} \rightarrow D \pi \pi$ may be responsible for a significant fraction of the observed decay widths.

The results for the calculated pion decay widths of the excited $D$ mesons obtained here are rather similar to those obtained in ref. [8], despite the different calculational framework and the different Hamiltonian model. The calculation in ref. [8] was restricted to the decays allowed by the lowest order selection rules suggested by heavy quark symmetry 10 . In the case of the $D_{1}$ meson the excluded $S$-wave transition was found to be rather important here, a result already hinted at in ref. [11]. The conclusion reached here is that the chiral quark model does indeed provide a fair description of the pion decay widths of the orbitally excited $D$ mesons, if they are treated as relativistic interacting two-quark systems.

This paper falls into 5 sections. In section 2 the decay width for $D^{*} \rightarrow D \pi$ is calculated. In section 3 the corresponding decay widths for the orbitally excited $D_{1}$ and the $D_{2}^{*}$ mesons, including the $S$-wave pion decay rate of the $D_{1}$ meson, are calculated. In section 4 the estimated pion decay widths of the hitherto undiscovered charm mesons with $L=1, J=1$ and $L=1, J=0$ are given. Section 5 contains a summarizing discussion. 


\section{Pion decay widths of the $D^{*}$ mesons}

\subsection{Single quark approximation}

The main contribution to the decay widths of the $D^{*}$ mesons in the ground state band is due to the pion decays $D^{*} \rightarrow D \pi$. These transitions are intriguing in that since the mass difference $M_{D^{*}}-M_{D}$ is very close to the pion mass, the available phase space is very small. Because of this closeness to the threshold for $\pi$ decay, and the nonzero mass splittings between the different charge states of the $\pi$ and $D$ mesons, one of these decays - the decay of the $D^{* 0}$ to $D^{ \pm} \pi^{\mp}$ - is in fact kinematically forbidden. The orbital wave functions of the constituents of the $D$ and $D^{*}$ mesons differ very little from one another, which implies that the main pionic decay mechanism is $P$-wave pion decay. Because of the consequent threshold suppression and the small phase space, the total widths of the $D^{*}$ mesons are expected to be very small. The current empirical upper bound for the total width of the $D^{* \pm}$ is $0.131 \mathrm{MeV}$ and that for the $D^{* 0}$ is $2.1 \mathrm{MeV}$ [12]. The former one of these upper bounds is already constraining for theoretical model calculations.

The $D$ and $D^{*}$ mesons are confined $q \bar{c}$ and $\bar{q} c$ systems, where $q$ and $\bar{q}$ denote constituent $u, d$ and $\bar{u}, \bar{d}$ quarks and $c$ and $\bar{c}$ charm and anticharm quarks respectively. The pions only couple to the light flavor quarks, the simplest model for the coupling being the chiral coupling:

$$
\mathcal{L}=i \frac{g_{A}^{q}}{2 f_{\pi}} \bar{\psi}_{q} \gamma_{5} \gamma_{\mu} \partial_{\mu} \vec{\phi}_{\pi} \cdot \vec{\tau} \psi_{q}
$$

Here $g_{A}^{q}$ denotes the axial coupling constant of the light flavor constituent quarks and $f_{\pi}$ is the pion decay constant $(93 \mathrm{MeV})$. The value of $g_{A}^{q}$ should be somewhere between unity [13] and $g_{A}^{q}=0.87$ [14, 15, 16. Only the $P$-wave part of the coupling (11) contributes to the pionic decays $D^{*} \rightarrow D \pi$, and hence, in the single quark approximation, the transition operator reduces to

$$
\begin{aligned}
T_{P} & =\frac{g_{A}^{q}}{2 f_{\pi}} \bar{u}\left(\vec{p}^{\prime}\right) \gamma_{5}^{q} \vec{\gamma}^{q} \cdot \vec{k} u(\vec{p}) \tau_{\pi} \\
& =-i \frac{g_{A}^{q}}{2 f_{\pi}} \sqrt{\frac{E^{\prime}+m}{2 E^{\prime}}} \sqrt{\frac{E+m}{2 E}}\left(1-\frac{P^{2}-k^{2} / 4}{3\left(E^{\prime}+m\right)(E+m)}\right) \vec{\sigma}^{q} \cdot \vec{k} \tau_{\pi} .
\end{aligned}
$$

Here $m$ denotes the mass of the light constituent quark and $\vec{k}$ is the momentum of the emitted pion. The operator $\vec{P}$ is defined as $\left(\vec{p}^{\prime}+\vec{p}\right) / 2$, with $\vec{p}$ and $\vec{p}^{\prime}$ being the initial and final momenta of the light quark. The energy factors $E$ and $E^{\prime}$ that appear in eq. (2) are defined as $\sqrt{p^{2}+m^{2}}$ and $\sqrt{p^{\prime 2}+m^{2}}$ respectively. The pionic decay widths of the $D^{*}$ mesons may now be expressed as

$$
\Gamma\left(D^{* 0} \rightarrow D^{0} \pi^{0}\right)=\frac{1}{24 \pi} \frac{E_{D^{0}}}{M_{D^{* 0}}}\left(\frac{g_{A}^{q}}{f_{\pi}}\right)^{2} k^{3} \mathcal{M}_{0}^{2}
$$

for the $D^{* 0}$ meson, and

$$
\begin{aligned}
\Gamma\left(D^{* \pm} \rightarrow D^{ \pm} \pi^{0}\right) & =\frac{1}{24 \pi} \frac{E_{D^{ \pm}}}{M_{D^{* \pm}}}\left(\frac{g_{A}^{q}}{f_{\pi}}\right)^{2} k^{3} \mathcal{M}_{0}^{2}, \\
\Gamma\left(D^{* \pm} \rightarrow D^{0} \pi^{ \pm}\right) & =\frac{1}{12 \pi} \frac{E_{D^{0}}}{M_{D^{* \pm}}}\left(\frac{g_{A}^{q}}{f_{\pi}}\right)^{2} k^{3} \mathcal{M}_{0}^{2}
\end{aligned}
$$


for the charged $D^{*}$ mesons. In the above expressions, $\mathcal{M}_{0}$ is the orbital part of the matrix element $\left\langle 00,00\left|\vec{\sigma}_{q} \cdot \vec{k}\right| 01,1 m\right\rangle$, where $|L S, J M\rangle$ denotes the state vector of the $q \bar{Q}$ system. To arrive at this expression, the following result for the sum over spins and integration over directions of $\vec{k}$ has been used:

$$
\frac{1}{3} \int d \Omega_{k} \sum_{m}\left\langle 1 m\left|\vec{\sigma}_{q} \cdot \vec{k}\right| 00\right\rangle\left\langle 00\left|\vec{\sigma}_{q} \cdot \vec{k}\right| 1 m\right\rangle=\frac{4 \pi}{3} k^{2} .
$$

Here $\vec{\sigma}_{q}$ denotes the spin operator of the light constituent quark. The matrix element $\mathcal{M}_{0}$ may be expressed in unapproximated form as

$$
\begin{aligned}
\mathcal{M}_{0}= & \frac{1}{\pi} \int_{0}^{\infty} d r^{\prime} r^{\prime} u_{0}\left(r^{\prime}\right) \int_{0}^{\infty} d r r u_{0}(r) \int_{0}^{\infty} d P P^{2} \int_{-1}^{1} d z f_{\mathrm{BS}}(P, z) \\
& \sqrt{\frac{E^{\prime}+m}{2 E^{\prime}}} \sqrt{\frac{E+m}{2 E}}\left(1-\frac{P^{2}-k^{2} / 4}{3\left(E^{\prime}+m\right)(E+m)}\right) \\
& j_{0}\left(r^{\prime} \sqrt{P^{2}+\frac{k^{2}}{16}+\frac{P k z}{2}}\right) j_{0}\left(r \sqrt{P^{2}+\frac{k^{2}}{16}-\frac{P k z}{2}}\right) .
\end{aligned}
$$

Here $u_{0}(r)$ is the reduced radial wave function for the $D$ and $D^{*}$ mesons. The explicit expressions for the energy factors appearing in eq. (7) are

$$
E=\sqrt{m^{2}+P^{2}-P k z+k^{2} / 4} \text { and } E^{\prime}=\sqrt{m^{2}+P^{2}+P k z+k^{2} / 4}
$$

respectively. The factor $f_{\mathrm{BS}}(P, z)$ originates in the quasipotential reduction of the amplitudes defined for the Bethe-Salpeter equation, and is defined as

$$
f_{\mathrm{BS}}(P, z)=\frac{M+m}{\sqrt{\left(E+E_{c}\right)\left(E^{\prime}+E_{c}^{\prime}\right)}} .
$$

Here $E_{c}=\sqrt{M^{2}+P^{2}-P k z+k^{2} / 4}$ and $E_{c}^{\prime}=\sqrt{M^{2}+P^{2}+P k z+k^{2} / 4}$ and $M$ is the heavy quark mass. In the non-relativistic limit the expression (7) reduces to

$$
\mathcal{M}_{0}=\int_{0}^{\infty} d r u_{0}^{2}(r) j_{0}\left(\frac{k r}{2}\right) .
$$

Because of the small masses of the light constituent quarks the non-relativistic approximation (10) turns out to be inadequate for a reliable description, and typically leads to overestimates of the exact result obtained from eq. (7) by at least a factor $\sim 2$. In order to evaluate the expression (7), a model for the wavefunctions is required. In this work, the wavefunctions that were obtained by solving the Blankenbecler-Sugar equation in configuration space in ref. [6] will be used. Those wavefunctions correspond to an interaction Hamiltonian which is a combination of relativistic one-gluon exchange and a scalar confining interaction. In this model the light quark mass was obtained as $450 \mathrm{MeV}$ and that of the charm quark as $1580 \mathrm{MeV}$. The string tension in the scalar confining interaction was taken to be $c=1120 \mathrm{MeV} / \mathrm{fm}$. The relativistic one-gluon exchange interaction employed in ref. [6] also features a running color "couplant", which was taken to be of the form [18]: 


$$
\alpha_{s}\left(k^{2}\right)=\frac{12 \pi}{27} \frac{1}{\ln \left[\frac{k^{2}+4 m_{g}^{2}}{\Lambda_{0}^{2}}\right]} .
$$

In eq. (11), $\Lambda_{0}$ denotes the confinement scale, for which the value $280 \mathrm{MeV}$ was used in ref. [6]. For the gluon mass parameter $m_{g}$, the value $m_{g}=240 \mathrm{MeV}$ was obtained. A comparison between the spectrum obtained in ref. [6] and the current empirical spectrum is shown in Fig. 11. The resulting reduced radial wave functions for the $1 S$ - and $1 P$-states that were obtained with this model in ref. [6] are shown in Fig. 2 .

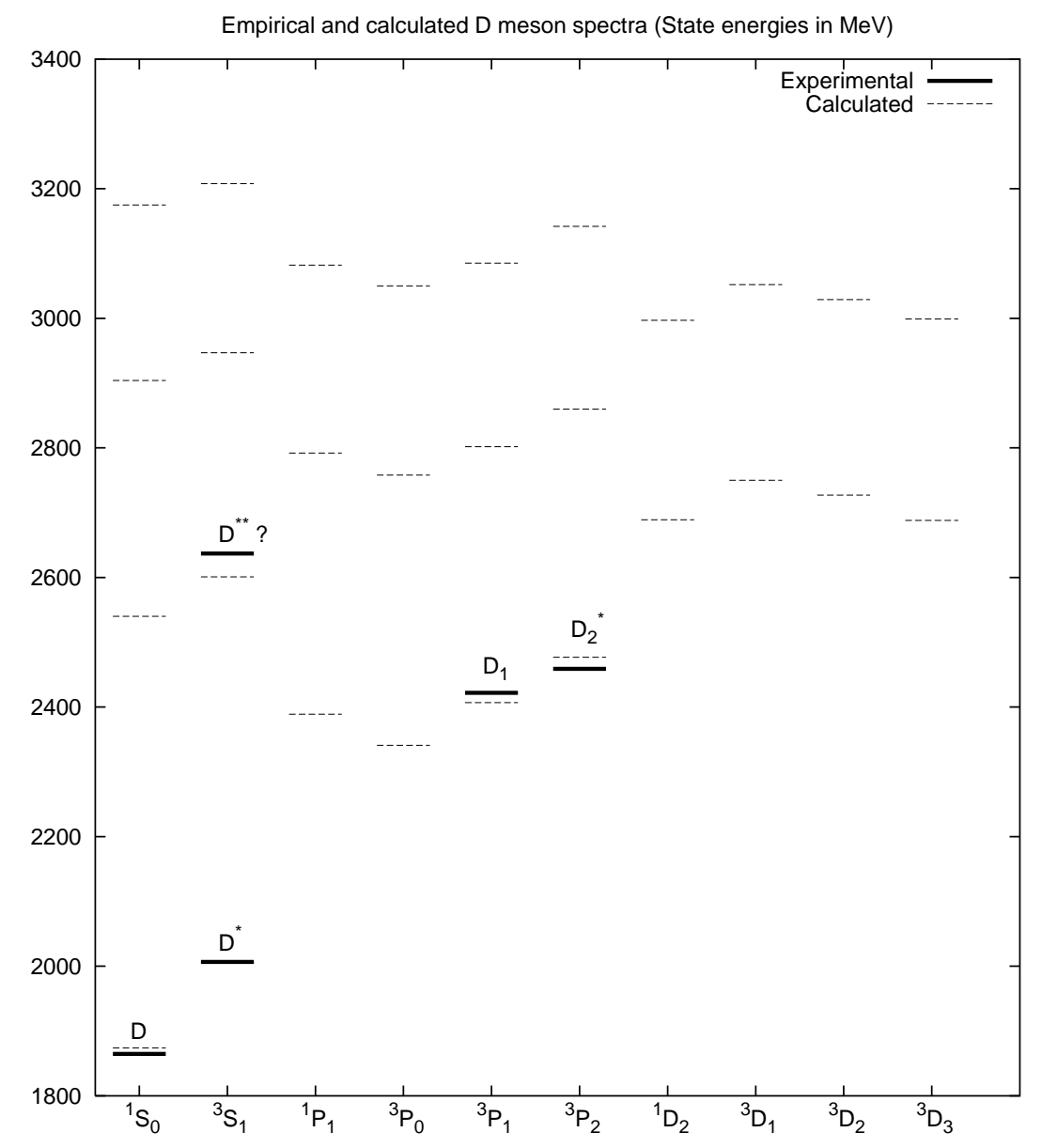

Figure 1: Experimental and calculated $D$ meson states. The calculated states are solutions to the Blankenbecler-Sugar equation and correspond to the Hamiltonian used in ref. [6]. The energies of the empirical states along with the pion momenta used in the calculations are listed in Table 1 . 


\begin{tabular}{l|c|c|c}
\multicolumn{1}{c|}{ Decay } & Initial state mass $\left(M_{i}\right)$ & Final state mass $\left(M_{f}\right)$ & $\pi$ momentum $(k)$ \\
\hline \hline & & & \\
$D^{* 0} \rightarrow D^{0} \pi^{0}$ & 2007 & 1865 & 43.1 \\
$D^{* \pm} \rightarrow D^{ \pm} \pi^{0}$ & 2010 & 1869 & 38.3 \\
$D^{* \pm} \rightarrow D^{0} \pi^{ \pm}$ & 2010 & 1865 & 39.6 \\
& & & \\
$D_{1} \rightarrow D^{*} \pi$ & 2422 & 2009 & 355 \\
$D_{2}^{*} \rightarrow D \pi$ & 2459 & 1867 & 505 \\
$D_{2}^{*} \rightarrow D^{*} \pi$ & 2459 & 2009 & 389 \\
$D_{1}^{*} \rightarrow D^{*} \pi$ & 2389 & 2009 & 326 \\
$D_{0}^{*} \rightarrow D \pi$ & 2341 & 1867 & 408
\end{tabular}

Table 1: Initial and final state $D$ meson masses and emitted pion momenta in $\mathrm{MeV}$ used in the calculations. When different charge states are specified, the values are taken from [12, otherwise suitable averages are used instead. The $D_{1}^{*}$ and $D_{0}^{*}$ masses are taken from ref. [6].

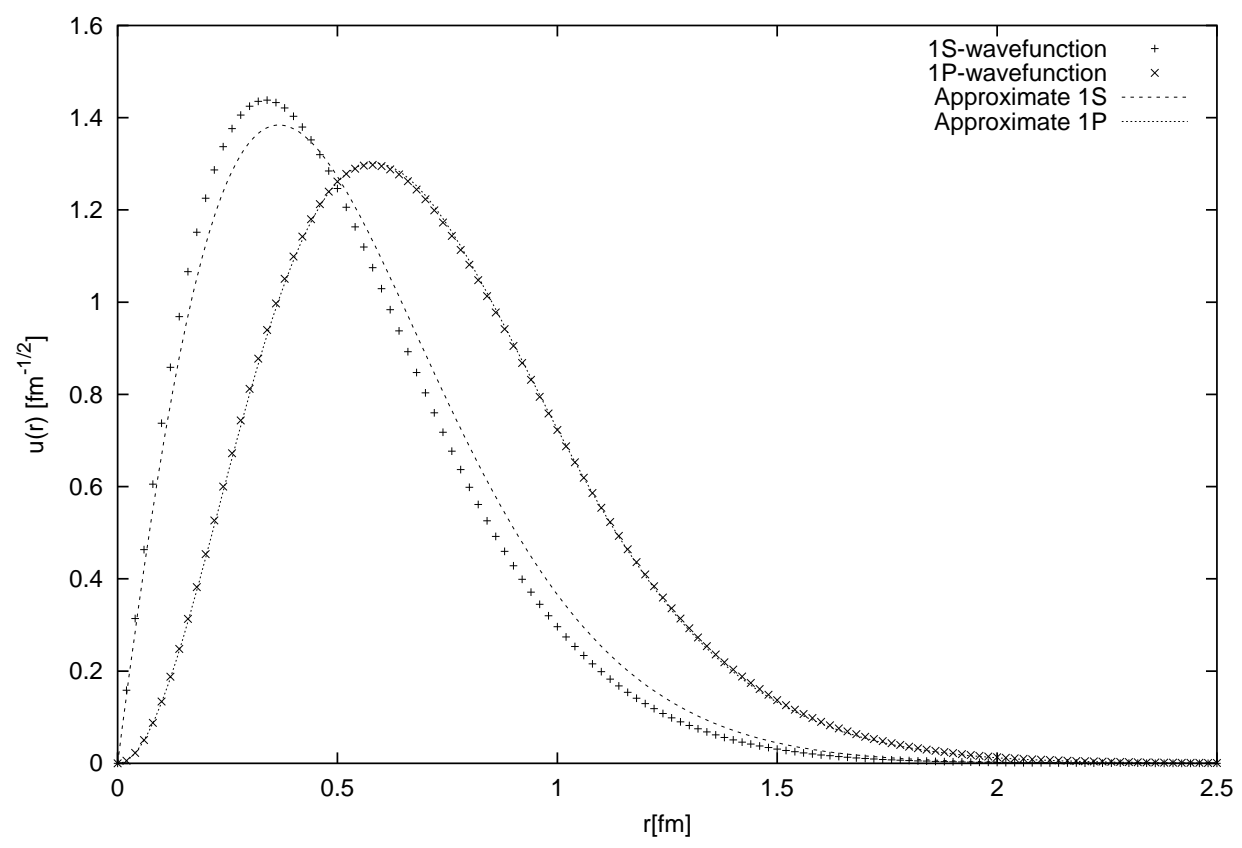

Figure 2: The reduced wave functions for the $S$ - and $P$-state $q \bar{c}$ (or $\bar{q} c$ ) systems according to ref. [6]. As these wavefunctions correspond to the spin-averaged $S$ - and $P$-states of the $D$ meson, they do not incorporate the fine structure splittings of the various states in Fig. 11. Therefore, in order to avoid unnecessary errors, the masses and pion momenta listed in Table 1 are used in the calculations. The approximate wavefunctions corresponding to eq. (55) in Appendix A are shown for comparison. 
The numerical value of the unapproximated matrix element $\mathcal{M}_{0}$, eq. (7), as obtained using the wave function $u_{0}(r)$ shown in Fig. 2 is $\mathcal{M}_{0}=0.649$. Although the value of the pion momentum $k$ in the decays of the $D^{*}$ mesons is non-negligible (In the case $D^{* \pm} \rightarrow D^{0} \pi^{+}$it is $39.6 \mathrm{MeV} / \mathrm{c}$ and in $D^{* 0} \rightarrow D^{0} \pi^{0}$ $43.1 \mathrm{MeV} / \mathrm{c})$ the product of this momentum and the range of the wave function $(\sim 0.5 \mathrm{fm})$, is of the order $\sim 0.1$. Because of the smallness of this product the value of the non-relativistic approximation to the matrix element $\mathcal{M}_{0}$ as evaluated from eq. 10 is $\simeq 1.0$. Thus one may conclude that even for the decays with the smallest pion momentum $k$, the non-relativistic approximation overestimates the calculated decay widths by about a factor 2.4 .

The calculated decay widths for $D^{*} \rightarrow D \pi$ as obtained with $g_{A}^{q}=0.87$ and $g_{A}^{q}=1$ are given in Table 2 along with the pion momenta used and the present empirical upper bounds. The $D$ meson masses used are those listed in Table 1. The calculated total pionic width of the $D^{* \pm}$ is $0.082 \mathrm{MeV}$ in the single quark approximation, a result, which is about $60 \%$ of the present empirical upper bound $(0.131 \mathrm{MeV})$. In the single quark approximation the calculated width of the $D^{* 0}$ is $0.036 \mathrm{MeV}$, which is far below the empirical upper bound of $1.3 \mathrm{MeV}$. In this case, the empirical upper bound is far too large for being theoretically constraining. The widths obtained here for the $D^{*}$ mesons are similar to those obtained in ref. [8], where the Gross equation framework was applied. In that reference, the value suggested for $g_{A}^{q}$ was however only 0.75 , and with that value the present calculated widths would be somewhat smaller. The difference may be attributed partly to the much lighter constituent quark masses used in ref. [8] and partly to the relativistic factors in eq. (7), which arise from the canonical boosts. Without those factors the calculated width of the $D^{* \pm}$ would exceed the experimental upper bound in the single quark approximation.

\subsection{Axial exchange current contribution}

The single quark amplitude, eq. (2), represents a coupling of the pion to the axial current of the light constituent quark, which in the static approximation may be expressed as

$$
\overrightarrow{A_{a}}=-g_{A}^{q} \vec{\sigma}_{q} \tau_{a} .
$$

The linear scalar confining interaction will contribute an exchange current that arises from coupling of the pion to a virtual negative energy quark, see Fig. 3. If the confining interaction is taken to be of the form $V_{c}(r)=c r-b$, as in ref. [6], then the expression for the corresponding exchange current operator may, to lowest order in $v / c$, be written as [17]:

$$
\vec{A}_{a}^{\mathrm{ex}}=-\frac{g_{A}^{q}}{4 m^{3}}\left[(c r-b)\left(3 \vec{\sigma}_{q} \vec{P}^{2}-4 \vec{P} \vec{\sigma}_{q} \cdot \vec{P}\right)+\frac{c}{2 r} \vec{\sigma}_{q}-\frac{2 c}{r} \vec{r} \times \vec{P}\right] \tau_{a} .
$$

This expression shows that the exchange current contribution is a relativistic correction of the same order in $v / c$ as the relativistic corrections to the single-quark operator in eq. (2). The constant $b$ was obtained as $320 \mathrm{MeV}$ in ref. [6].

The expression (13) does not include the corrections from the canonical boost factors on the single quark spinors that are included in the single quark operator, eq. (2). Hence a more realistic evaluation requires that those factors are taken into account. For simplicity, the same spinor factors as for the single quark operator are used in this context, and the operator is also multiplied with the corresponding

- numerically less important - spinor normalization factors for the scalar vertex on the heavy antiquark line. This implies going beyond the static local approximation for the confining potential. Accordingly the potential function $c r$ is to be replaced with the general expression $c\left|\vec{r}^{\prime}+\vec{r}\right| / 2$. 
Moreover the factor $1 / \mathrm{m}^{3}$ in the axial exchange current operator (12) arises as the static approximation to a combination of energy dependent factors appearing in the denominator for the intermediate negative energy state and of corresponding energy factors in the quark spinors. Consequently, the static approximation to these factors implies a very large overestimate of the axial exchange current contribution. In order to obtain a more realistic estimate for this contribution, the static approximation in the axial exchange current operator may be replaced as

$$
\frac{1}{m^{3}} \rightarrow \frac{4}{2 m+E+E^{\prime}}\left(\frac{2 m}{E+E^{\prime}}\right)^{2} .
$$

The resulting matrix element of the exchange current operator (13) between the triplet and singlet states may then be expressed as

$$
\begin{aligned}
\mathcal{M}_{0}^{\mathrm{ex}}= & \frac{5}{12 \pi} \int_{0}^{\infty} d r^{\prime} r^{\prime} u_{0}\left(r^{\prime}\right) \int_{0}^{\infty} d r r u_{0}(r) V_{c}\left(\sqrt{\frac{r^{\prime 2}+r^{2}}{2}}\right) \int_{0}^{\infty} d P P^{4} \\
& \int_{-1}^{1} d z f_{\mathrm{BS}}(P, z) j_{0}\left(r^{\prime} \sqrt{P^{2}+\frac{k^{2}}{16}+\frac{P k z}{2}}\right) j_{0}\left(r \sqrt{P^{2}+\frac{k^{2}}{16}-\frac{P k z}{2}}\right) \\
& \frac{4}{2 m+E+E^{\prime}}\left(\frac{2}{E+E^{\prime}}\right)^{2} \sqrt{\frac{E^{\prime}+m}{2 E^{\prime}}} \sqrt{\frac{E+m}{2 E}}\left(1-\frac{P^{2}-k^{2} / 4}{3\left(E^{\prime}+m\right)(E+m)}\right) \\
& \sqrt{\frac{E_{c}^{\prime}+M}{2 E_{c}^{\prime}}} \sqrt{\frac{E_{c}+M}{2 E_{c}}}\left(1-\frac{P^{2}-k^{2} / 4}{\left(E_{c}^{\prime}+M\right)\left(E_{c}+M\right)}\right) .
\end{aligned}
$$

In the above expression, a symmetrized form $\sqrt{\left(r^{\prime 2}+r^{2}\right) / 2}$ of the confining potential has been employed for simplicity. This form leads to the correct limit when $r=r^{\prime}$. Moreover, the term proportional to $c / 2 r$ in eq. (13) has been neglected in the calculations because of its smallness. The numerical value of the exchange current matrix element $\mathcal{M}_{0}^{\text {ex }}$ as evaluated using eq. (15) is obtained as 0.040 . In the static limit, i.e. without the replacement of eq. (14), that value increases by about a factor 3 . The static limit is thus seen to represent a significant overestimate of this effect. In any case, the correction is non-negligible in comparison with the contribution from the single quark operator, which is $\mathcal{M}_{0}=0.649$. Addition of the exchange current contribution increases the calculated pionic decay widths by about $15 \%$, and brings them to the empirical upper limits in the case of the charged $D^{*}$ mesons, if $g_{A}^{q}=1$. These results are also shown in Table 2.

Even though the total widths of the $D^{* \pm}$ states have not yet been experimentally determined, their branching fractions for $\pi$ decay are known to a good degree of accuracy. The current experimental results are $(68.3 \pm 1.4) \%$ for $D^{* \pm} \rightarrow D^{0} \pi^{ \pm}$and $(30.6 \pm 2.5) \%$ for $D^{* \pm} \rightarrow D^{ \pm} \pi^{0}$ respectively [12. Using the results in Table 2, the ratio of these decay modes is obtained as

$$
\frac{\Gamma\left(D^{* \pm} \rightarrow D^{0} \pi^{ \pm}\right)}{\Gamma\left(D^{* \pm} \rightarrow D^{ \pm} \pi^{0}\right)}=2.2
$$

being thus in excellent agreement both with the result obtained in ref. [8] and the experimentally determined value $2.23 \pm 0.19$. 


\begin{tabular}{l|c|c|c|c|c} 
Decay & $\pi$ momentum $k$ & $\mathrm{SQA}$ & $\mathrm{SQA}+\mathrm{EXCH}$ & $g_{A}^{q}=1$ & Experiment \\
\hline \hline$D^{* \pm} \rightarrow D^{ \pm} \pi^{0}$ & 38.3 & 0.026 & 0.029 & 0.038 & $<0.04$ \\
$D^{* \pm} \rightarrow D^{0} \pi^{ \pm}$ & 39.6 & 0.056 & 0.064 & 0.084 & $<0.09$ \\
& & & & & \\
$D^{* 0} \rightarrow D^{0} \pi^{0}$ & 43.1 & 0.036 & 0.041 & 0.054 & $<1.3$
\end{tabular}

Table 2: The calculated pionic widths and experimental upper limits in $\mathrm{MeV}$ for the $D^{*}$ mesons, corresponding to $g_{A}^{q}=0.87$. The single quark approximation, with relativistic corrections is denoted SQA, and the result obtained with the exchange current contribution included is denoted SQA + EXCH. The net calculated widths are also shown for $g_{A}^{q}=1$.

\section{Pion decay widths of the $D_{1}$ and $D_{2}^{*}$ mesons}

\subsection{Pion decay by the single quark axial current}

Only two of the four expected charm meson resonances with $L=1$ have hitherto been discovered with certainty. These are the $D_{1}(2420)$ and the $D_{2}^{*}(2460)$ mesons, including all the different charge states. It is generally assumed that these are spin triplet states with $J=1$ and $J=2$ respectively. In the Heavy Quark Symmetry (HQS) framework, they are assumed to be states with light quark angular momentum $j_{q}=3 / 2$. For these $D$ meson states the total widths have been experimentally determined, although with quite large uncertainty ranges, and some of their pionic decays have been "seen" [12]. The basic pionic decay mode of these resonances is $D$-wave decay by pion coupling to the axial current operator, eq. (2), of the charm mesons. In the case of the $D_{1}$ meson, $S$-wave pion decay through the axial charge operator also contributes significantly to the decay width [11].

The axial current transition operator for pion decay to the ground state $D$ mesons is given by eq. (2). If both the $D_{1}(2420)$ and $D_{2}^{*}(2460)$ mesons are assumed to be mainly spin triplet states, the calculation of the pion decay widths of these states require the following spin sums:

$$
\begin{aligned}
S_{s} & =\frac{1}{2 J+1} \sum_{M=-J}^{J}\left\langle 11, J M\left|\vec{\sigma}_{q} \cdot \vec{k}\right| 00,00\right\rangle\left\langle 00,00\left|\vec{\sigma}_{q} \cdot \vec{k}\right| 11, J M\right\rangle \\
& =\frac{1}{2 J+1} \sum_{M=-J}^{J}\left\langle 11, J M\left|\frac{1}{3} k^{2}-\frac{1}{6} S_{12}(\vec{k})\right| 11, J M\right\rangle,
\end{aligned}
$$

for spin singlet final states, and

$$
\begin{aligned}
S_{t} & =\frac{1}{2 J+1} \sum_{M=-J}^{J} \sum_{m=-1}^{1}\left\langle 11, J M\left|\vec{\sigma}_{q} \cdot \vec{k}\right| 01,1 m\right\rangle\left\langle 01,1 m\left|\vec{\sigma}_{q} \cdot \vec{k}\right| 11, J M\right\rangle \\
& =\frac{1}{2 J+1} \sum_{M=-J}^{J}\left\langle 11, J M\left|\frac{2}{3} k^{2}+\frac{1}{6} S_{12}(\vec{k})\right| 11, J M\right\rangle,
\end{aligned}
$$


for spin triplet final states. In the above expressions, $S_{12}$ denotes the tensor operator

$$
S_{12}(\hat{k})=3 \vec{\sigma}_{q} \cdot \hat{k} \vec{\sigma}_{\bar{Q}} \cdot \hat{k}-\vec{\sigma}_{q} \cdot \vec{\sigma}_{\bar{Q}} .
$$

Evaluation of the orbital matrix elements of these operators then leads to the following expressions for pion decay driven by the axial current operator:

$$
\begin{aligned}
\Gamma_{A}\left(D_{1} \rightarrow D \pi\right) & =0, \\
\Gamma_{A}\left(D_{1} \rightarrow D^{*} \pi\right) & =\frac{3}{8 \pi} \frac{E_{D^{*}}}{M_{D_{1}}}\left(\frac{g_{A}^{q}}{f_{\pi}}\right)^{2} k^{3} \mathcal{M}_{1}^{2},
\end{aligned}
$$

for the $D_{1}$ meson, and

$$
\begin{aligned}
\Gamma_{A}\left(D_{2}^{*} \rightarrow D \pi\right) & =\frac{3}{8 \pi} \frac{2}{5} \frac{E_{D}}{M_{D_{2}^{*}}}\left(\frac{g_{A}^{q}}{f_{\pi}}\right)^{2} k^{3} \mathcal{M}_{1}^{2}, \\
\Gamma_{A}\left(D_{2}^{*} \rightarrow D^{*} \pi\right) & =\frac{3}{8 \pi} \frac{3}{5} \frac{E_{D^{*}}}{M_{D_{2}^{*}}}\left(\frac{g_{A}^{q}}{f_{\pi}}\right)^{2} k^{3} \mathcal{M}_{1}^{2} .
\end{aligned}
$$

for the $D_{2}^{*}$ meson. In the above expressions, all final charge states have been included. $\mathcal{M}_{1}$ is an orbital matrix element defined in analogy with eq. ( 7 and may be expressed as

$$
\begin{aligned}
\mathcal{M}_{1}= & \frac{1}{\pi} \int_{0}^{\infty} d r^{\prime} r^{\prime} u_{0}\left(r^{\prime}\right) \int_{0}^{\infty} d r r u_{1}(r) \int_{0}^{\infty} d P P^{2} \int_{-1}^{1} d z f_{\mathrm{BS}}(P, z) \\
& \sqrt{\frac{E^{\prime}+m}{2 E^{\prime}}} \sqrt{\frac{E+m}{2 E}}\left(1-\frac{P^{2}-k^{2} / 4}{3\left(E^{\prime}+m\right)(E+m)}\right) \frac{k / 4-P z}{\sqrt{P^{2}+k^{2} / 16-P k z / 2}} \\
& j_{0}\left(r^{\prime} \sqrt{P^{2}+\frac{k^{2}}{16}+\frac{P k z}{2}}\right) j_{1}\left(r \sqrt{P^{2}+\frac{k^{2}}{16}-\frac{P k z}{2}}\right) .
\end{aligned}
$$

In eq. (24), the factor $f_{\mathrm{BS}}(P, z)$ is defined as in eq. (9), and the quark energy factors $E, E^{\prime}$ are defined as for eq. (7). $u_{1}(r)$ is the reduced radial wave function for the $D$ meson $P$-state

$$
|11, J M\rangle=\sum_{l s}\left\langle 11 l s|J M| \frac{u_{1}(r)}{r} Y_{1 l}(\hat{r}) \mid 1 s\right\rangle,
$$

where $|1 s\rangle$ denotes a spin triplet state with $s_{z}=s$. In the non-relativistic limit the matrix element (24) reduces to

$$
\mathcal{M}_{1}=\int_{0}^{\infty} d r u_{0}(r) u_{1}(r) j_{1}\left(\frac{k r}{2}\right) .
$$

Note that eq. (20) predicts that the $D_{1}$ meson cannot decay to $D \pi$. This prediction only holds if the $D_{1}$ is a state with $J=1$. As will be shown, it does not provide a means to discriminate between spin singlet and triplet states. However, the prediction that the $D_{2}^{*}$ decays to both $D^{*}$ and $D$ permits 
the identification of the empirical $D_{2}^{*}(2460)$ meson as a spin triplet state with $J=2$. The numerical values for the matrix element $\mathcal{M}_{1}$, as obtained with the $L=0$ and $L=1$ wave functions shown in Fig. 2 for the pionic decay modes (21-23), are listed in Table 3. In all cases, the non-relativistic approximation overestimates the calculated matrix elements by $\sim 50 \%$, which is to be expected in view of the relatively small masses of the light constituent quarks. The calculated pionic decay widths corresponding to the matrix elements in Table 3 are given in Table 5 along with the current empirical values.

\subsection{Axial exchange current contribution}

The axial exchange current operator, eq. (13), may also contribute significantly to the pionic decay widths of the $L=1$ charm mesons. The orbital matrix element of the spin part of the exchange current operator between the $L=1$ and $L=0$ states, when evaluated with the same spinor factors and approximations as in eq. (15), may be expressed as

$$
\begin{aligned}
\mathcal{M}_{1}^{\mathrm{ex}}= & \frac{5}{12 \pi} \int_{0}^{\infty} d r^{\prime} r^{\prime} u_{0}\left(r^{\prime}\right) \int_{0}^{\infty} d r r u_{1}(r) V_{c}\left(\sqrt{\frac{r^{\prime 2}+r^{2}}{2}}\right) \int_{0}^{\infty} d P P^{4} \int_{-1}^{1} d z f_{\mathrm{BS}}(P, z) \\
& j_{0}\left(r^{\prime} \sqrt{P^{2}+\frac{k^{2}}{16}+\frac{P k z}{2}}\right) j_{1}\left(r \sqrt{P^{2}+\frac{k^{2}}{16}-\frac{P k z}{2}}\right) \frac{k / 4-P z}{\sqrt{P^{2}+k^{2} / 16-P k z / 2}} \\
& \frac{4}{2 m+E+E^{\prime}}\left(\frac{2}{E+E^{\prime}}\right)^{2} \sqrt{\frac{E^{\prime}+m}{2 E^{\prime}}} \sqrt{\frac{E+m}{2 E}}\left(1-\frac{P^{2}-k^{2} / 4}{3\left(E^{\prime}+m\right)(E+m)}\right) \\
& \sqrt{\frac{E_{c}^{\prime}+M}{2 E_{c}^{\prime}}} \sqrt{\frac{E_{c}+M}{2 E_{c}}}\left(1-\frac{P^{2}-k^{2} / 4}{\left(E_{c}^{\prime}+M\right)\left(E_{c}+M\right)}\right) .
\end{aligned}
$$

The numerical values of these matrix elements are also listed in Table 3. Their magnitude corresponds to $\sim 10 \%$ of the matrix elements of the corresponding single quark operator, eq. (24), and evidently the exchange current contribution is numerically significant these cases as well. This contribution enhances the net calculated pionic decay widths of the $L=1$ charm mesons and brings them closer to the empirical values. These results are shown in Table 5 .

\begin{tabular}{c|c|c|c} 
Decay & SQA-NR & SQA-REL & EXCH \\
\hline \hline$D_{1} \rightarrow D^{*} \pi$ & 0.135 & 0.093 & 0.010 \\
& & & \\
$D_{2}^{*} \rightarrow D \pi$ & 0.185 & 0.126 & 0.012 \\
& & & \\
$D_{2}^{*} \rightarrow D^{*} \pi$ & 0.147 & 0.101 & 0.011
\end{tabular}

Table 3: Matrix elements $\mathcal{M}_{1}$ of the single quark axial current operator in the non-relativistic approximation, eq. (26), and without approximation, eq. (24), for pion decay of the $D_{1}$ and $D_{2}^{*}$ mesons. The column "exchange" contains the matrix elements $\mathcal{M}_{1}^{\text {ex }}$ corresponding to eq. (27) of the spin part of the axial exchange current operator (13). 


\subsection{Pion decay by the axial charge}

The charge component of the axial vector coupling in eq. (1) also contributes to the decays of the $D$-mesons with $L=1$, and gives rise to both $S$ - and $D$-wave pion decay. The amplitude describing the coupling of the pion field to the axial charge component of the light constituent quark may then be obtained from eq. (11) as

$$
T_{S}=i \frac{g_{A}^{q}}{2 f_{\pi}} \frac{2 m+E+E^{\prime}}{\sqrt{4 E E^{\prime}(E+m)\left(E^{\prime}+m\right)}} \omega_{\pi} \vec{\sigma} \cdot\left(\frac{\vec{p}^{\prime}+\vec{p}}{2}\right) \tau_{\pi}
$$

where $\omega_{\pi}=\sqrt{k^{2}+m_{\pi}^{2}}$ is the energy of the emitted pion. In the evaluation of the matrix element of $T_{S}$ between $D$-meson states with $L=1$ and the ground state charm mesons, the operator $\left(\vec{p}^{\prime}+\vec{p}\right) / 2$ is treated as the differential operator $i\left(\vec{\nabla}^{\prime}-\vec{\nabla}\right) / 2$. Application of the operators on the initial and final orbital wave functions leads to spin matrix elements of the form

$$
\left\langle S^{\prime} M\left|\sum_{l s}\langle 11 l s \mid J M\rangle \sigma_{l}^{1}\right| 1 s\right\rangle=-\delta_{J S^{\prime}} \delta_{M^{\prime} M}\left[\sqrt{3} \delta_{S^{\prime} 0}+\sqrt{2} \delta_{S^{\prime} 1}\right],
$$

from which it follows that $S$-wave pion decay can only contribute to the $D_{1} \rightarrow D^{*} \pi$ decay widths. The resulting contribution to the $D_{1}(2420) \rightarrow D^{*} \pi$ decay width from $S$-wave pion decay may be written in the form

$$
\Gamma_{C}\left(D_{1} \rightarrow D^{*} \pi\right)=\frac{1}{32 \pi} \frac{E_{D^{*}}}{M_{D_{1}}}\left(\frac{g_{A}^{q}}{f_{\pi}}\right)^{2}\left(\frac{\omega_{\pi}}{m}\right)^{2} k \mathcal{M}_{1 \mathrm{~S}}^{2}
$$

In eq. (30), the matrix element $\mathcal{M}_{1 \mathrm{~S}}$ is defined, in analogy with the matrix elements in eqs. (7) and (24), as

$$
\begin{aligned}
\mathcal{M}_{1 \mathrm{~S}}= & \frac{1}{\pi} \int_{0}^{\infty} d r^{\prime} r^{\prime} \int_{0}^{\infty} d r r \int_{0}^{\infty} d P P^{2} \int_{-1}^{1} d z f_{\mathrm{BS}}(P, z) \\
& \frac{m\left(2 m+E+E^{\prime}\right)}{\sqrt{4 E E^{\prime}(E+m)\left(E^{\prime}+m\right)}}\left[u_{0}^{\prime}\left(r^{\prime}\right) u_{1}(r)-u_{0}\left(r^{\prime}\right) u_{1}^{\prime}(r)-2 \frac{u_{0}\left(r^{\prime}\right) u_{1}(r)}{r}\right] \\
& j_{0}\left(r^{\prime} \sqrt{P^{2}+\frac{k^{2}}{16}+\frac{P k z}{2}}\right) j_{0}\left(r \sqrt{P^{2}+\frac{k^{2}}{16}-\frac{P k z}{2}}\right)
\end{aligned}
$$

In the above expression, the factors $u^{\prime}(r)$ denote the derivatives of the reduced radial wavefunctions displayed in Fig. 2. In the non-relativistic limit, the matrix element (31) simplifies to

$$
\mathcal{M}_{1 \mathrm{~S}}=\int_{0}^{\infty} d r\left[u_{0}^{\prime}(r) u_{1}(r)-u_{0}(r) u_{1}^{\prime}(r)-2 \frac{u_{0}(r) u_{1}(r)}{r}\right] j_{0}\left(\frac{k r}{2}\right) .
$$

The numerical values of these matrix elements are given in Table $\square$. The values of the matrix elements $\mathcal{M}_{1 \mathrm{~S}}$ in Table 1 are very large and lead to unrealistically large contributions from $S$-wave pion decay to the widths of the $D_{1}$ mesons. Insertion in eq. (30) would give for $\Gamma_{C}\left(D_{1} \rightarrow D^{*} \pi\right)$ the value $\sim 60$ $\mathrm{MeV}$, which exceeds the empirically determined width by about a factor 4 . Reduction of this value to a realistic level is however brought about by the two-body mechanism described in Section 3.4 that is implied by the linear confining interaction, if its spinor structure is scalar. 
The amplitude (28) also gives rise to $D$-wave pion decay, which contributes to the decay widths of both the $D_{1}$ and $D_{2}^{*}$ mesons. The explicit expressions for these contributions from $D$-wave pion decay may be obtained as

$$
\Gamma_{D}\left(D_{1} \rightarrow D^{*} \pi\right)=\frac{1}{8 \pi} \frac{E_{D^{*}}}{M_{D_{1}}}\left(\frac{g_{A}^{q}}{f_{\pi}}\right)^{2}\left(\frac{\omega_{\pi}}{m}\right)^{2} k \mathcal{M}_{1 \mathrm{D}}^{2}
$$

for the $D_{1}$ meson, and

$$
\begin{aligned}
\Gamma_{D}\left(D_{2}^{*} \rightarrow D \pi\right) & =\frac{3}{8 \pi} \frac{2}{5} \frac{E_{D}}{M_{D_{2}^{*}}}\left(\frac{g_{A}^{q}}{f_{\pi}}\right)^{2}\left(\frac{\omega_{\pi}}{m}\right)^{2} k \mathcal{M}_{1 \mathrm{D}}^{2} \\
\Gamma_{D}\left(D_{2}^{*} \rightarrow D^{*} \pi\right) & =\frac{3}{8 \pi} \frac{3}{5} \frac{E_{D^{*}}}{M_{D_{2}^{*}}}\left(\frac{g_{A}^{q}}{f_{\pi}}\right)^{2}\left(\frac{\omega_{\pi}}{m}\right)^{2} k \mathcal{M}_{1 \mathrm{D}}^{2}
\end{aligned}
$$

for the $D_{2}^{*}$ meson. In the above expressions, the matrix element $\mathcal{M}_{1 \mathrm{D}}$ is again defined analogously to eq. (31), and may be expressed as

$$
\begin{aligned}
\mathcal{M}_{1 \mathrm{D}}= & -\frac{1}{\pi} \int_{0}^{\infty} d r^{\prime} r^{\prime} \int_{0}^{\infty} d r r \int_{0}^{\infty} d P P^{2} \int_{-1}^{1} d z f_{\mathrm{BS}}(P, z)\left(\frac{3}{2} \frac{P^{2}\left(1-z^{2}\right)}{P^{2}+\frac{k^{2}}{16}-\frac{P k z}{2}}-1\right) \\
& \frac{m\left(2 m+E+E^{\prime}\right)}{\sqrt{4 E E^{\prime}(E+m)\left(E^{\prime}+m\right)}}\left[u_{0}^{\prime}\left(r^{\prime}\right) u_{1}(r)-u_{0}\left(r^{\prime}\right) u_{1}^{\prime}(r)+\frac{u_{0}\left(r^{\prime}\right) u_{1}(r)}{r}\right] \\
& j_{0}\left(r^{\prime} \sqrt{P^{2}+\frac{k^{2}}{16}+\frac{P k z}{2}}\right) j_{2}\left(r \sqrt{P^{2}+\frac{k^{2}}{16}-\frac{P k z}{2}}\right) .
\end{aligned}
$$

In the non-relativistic limit, this expression reduces to

$$
\mathcal{M}_{1 \mathrm{D}} \simeq \int_{0}^{\infty} d r\left[u_{0}^{\prime}(r) u_{1}(r)-u_{0}(r) u_{1}^{\prime}(r)+\frac{u_{0}(r) u_{1}(r)}{r}\right] j_{2}\left(\frac{k r}{2}\right) .
$$

The numerical values of these matrix elements for $D$-wave decay as obtained with the wavefunctions of ref. [6] turn out to be exceedingly tiny in comparison with those from $S$-wave decay. The reason for this is immediately apparent if one considers the wave function combination $u_{0}^{\prime} u_{1}-u_{0} u_{1}^{\prime}+u_{0} u_{1} / r$ that appears in the integrand in eq. (37). If the approximate wave functions plotted in Fig. 2 and given in Appendix A are used, that wave function combination vanishes exactly. As these approximate wavefunctions are not perfect and as the nonlocal structure of the integrand in eq. (36) has to be taken into account, this cancellation is not absolute if the unapproximated expressions and wave functions are used. The numerical values of eqs. (36) and (37) turn out to be of the order $1 \mathrm{MeV}$ and thus completely negligible as compared to the matrix elements of $S$-wave decay listed in Table 4 . These $D$-wave decay amplitudes that arise from the axial charge operator also obtain a contribution from the two-body mechanism outlined in Section 3.4 . 


\subsection{Two-quark contributions to the axial charge operator}

Both the confining and one-gluon exchange (OGE) interactions contribute two-body terms to the axial charge density operator. If the linear confining interaction has the form $V_{c}(r) \mathcal{S}$, where $V_{c}$ is the central confining potential, and $\mathcal{S}$ is the scalar (Fermi) invariant for the two-quark system, it gives rise to an axial exchange charge density operator, which to lowest order in $v / c$ may be expressed as 19

$$
A_{\mathrm{conf}}^{0}=\frac{g_{A}^{q}}{m^{2}} V_{c}(r) \vec{\sigma} \cdot \vec{P} \tau_{a} .
$$

The corresponding expression for the vector coupled one-gluon exchange interaction is

$$
A_{\mathrm{OGE}}^{0}=\frac{g_{A}^{q}}{m M} V_{g}(r) \vec{\sigma} \cdot \vec{P}_{\bar{c}} \tau_{a},
$$

where the momentum operator $\vec{P}_{\bar{c}}$ is defined as $\vec{P}_{\bar{c}}=\left(\vec{p}_{\bar{c}}{ }^{\prime}+\vec{p}_{\bar{c}}\right) / 2$. Here $\vec{p}_{\bar{c}}$ and $\vec{p}_{\bar{c}}{ }^{\prime}$ denote the momentum operators of the heavy quark and $V_{g}(r)$ is the main spin-independent term in the one-gluon exchange potential. If the color couplant $\alpha_{s}$ is taken to be constant, then $V_{g}(r)$ may be expressed as $V_{g}(r)=$ $-4 \alpha_{s} / 3 r$ in the static limit.
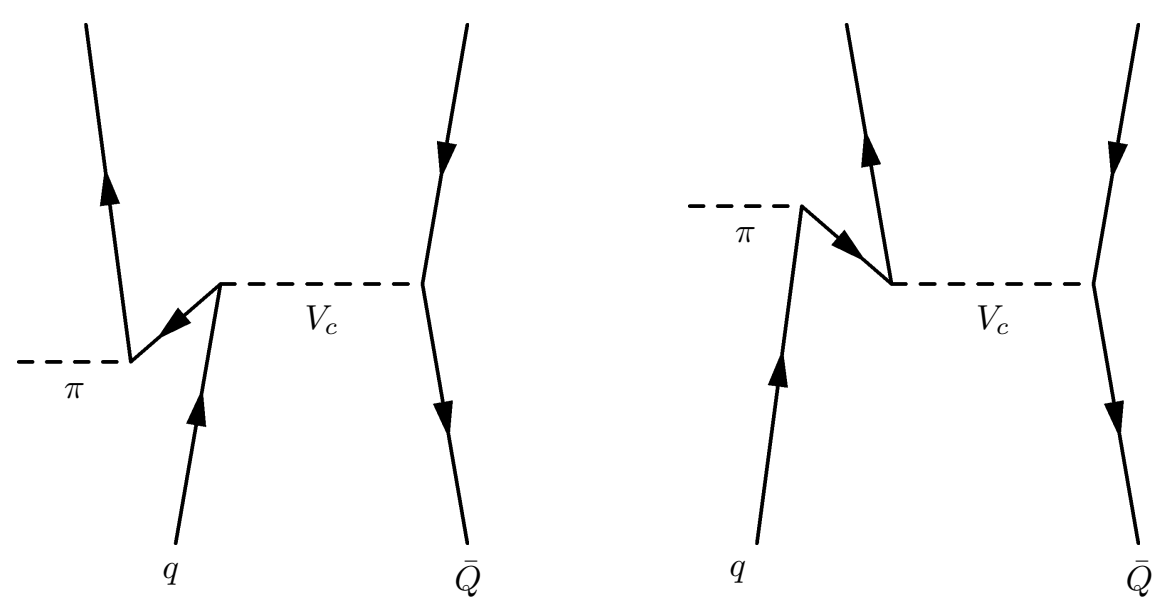

Figure 3: Two-quark contributions to the pion production amplitude associated with the axial charge operator. The diagrams shown correspond to both time orderings of the two-quark contribution from the scalar confining interaction. Two similar diagrams arise from the one-gluon exchange interaction, in which case the scalar vertices are to be appropriately replaced.

In the static limit, the two-body contribution that arises from the confining and one-gluon exchange interactions to the amplitude for $S$-wave pion decay may be expressed in the form

$$
T_{S}^{(2)}=-\frac{1}{m}\left(c r-b+\frac{4}{3} \frac{m}{M} \frac{\alpha_{s}}{r}\right) T_{S}
$$


Here, $T_{S}$ denotes the single quark amplitude for $S$-wave pion decay, eq. (28). This result may be derived as a pair current operator, or more simply by making the scalar shift in the light constituent quark mass $m \rightarrow m+V_{c}(r)$ in the case of the scalar confining interaction. This two-body operator arises in the non-relativistic reduction of the general pion decay amplitude for the $q \bar{Q}$ system as a pair (or seagull) term, see Fig. 3. A corresponding two-body term appears in the amplitude for pion production in nucleon-nucleon collisions, the difference being that $V_{c}$ is in that case replaced by the effective scalar component of the nucleon-nucleon interaction.

In eq. (40) the factor $1 / \mathrm{m}$ represents the static limit of the propagator of the intermediate negative energy quark. As the static limit is known, from the treatment of the axial exchange current, to give large overestimates in the case of light constituent quarks, the static propagator will here be replaced by the symmetrized form $4 /\left(2 m+E+E^{\prime}\right)$. The matrix element of the two-quark contribution to the $S$-wave decays through the axial charge operator may then be obtained by modifying the single quark matrix element for $S$-wave pion decay, eq. (31), according to eq. (40), giving

$$
\begin{aligned}
\mathcal{M}_{2 \mathrm{~S}}^{\mathrm{conf}} \simeq & -\frac{1}{\pi} \int_{0}^{\infty} d r^{\prime} r^{\prime} \int_{0}^{\infty} d r r V_{c}\left(\sqrt{\frac{r^{2}+r^{\prime 2}}{2}}\right) \int_{0}^{\infty} d P P^{2} \int_{-1}^{1} d z f_{\mathrm{BS}}(P, z) \\
& \frac{4 m}{\sqrt{4 E E^{\prime}(E+m)\left(E^{\prime}+m\right)}}\left[u_{0}^{\prime}\left(r^{\prime}\right) u_{1}(r)-u_{0}\left(r^{\prime}\right) u_{1}^{\prime}(r)-2 \frac{u_{0}\left(r^{\prime}\right) u_{1}(r)}{r}\right] \\
& j_{0}\left(r^{\prime} \sqrt{P^{2}+\frac{k^{2}}{16}+\frac{P k z}{2}}\right) j_{0}\left(r \sqrt{P^{2}+\frac{k^{2}}{16}-\frac{P k z}{2}}\right) \\
& \sqrt{\frac{E_{c}^{\prime}+M}{2 E_{c}^{\prime}}} \sqrt{\frac{E_{c}+M}{2 E_{c}}}\left(1-\frac{P^{2}-k^{2} / 4}{\left(E_{c}^{\prime}+M\right)\left(E_{c}+M\right)}\right) .
\end{aligned}
$$

In the above expression, the symbol $\simeq$ indicates that $\left|\vec{r}+\vec{r}^{\prime}\right| / 2$ has been approximated by the expression $\sqrt{\left(r^{\prime 2}+r^{2}\right) / 2}$ as in eq. (15). In the nonrelativistic limit, eq. (41) becomes

$$
\mathcal{M}_{2 \mathrm{~S}}^{\mathrm{conf}} \simeq-\frac{1}{m} \int_{0}^{\infty} d r V_{c}(r)\left[u_{0}^{\prime}(r) u_{1}(r)-u_{0}(r) u_{1}^{\prime}(r)-2 \frac{u_{0}(r) u_{1}(r)}{r}\right] j_{0}\left(\frac{k r}{2}\right) .
$$

The gluon exchange contribution, eq. (39), to the axial exchange charge operator gives a contribution of smaller magnitude than the confining interaction because of the heavy antiquark mass involved. Since the heavy quark constituent mass, $1580 \mathrm{MeV}$, is not very large compared to the light quark mass of 450 $\mathrm{MeV}$, the one-gluon exchange contribution turns out to be significant as well, see Table $\mathbb{E}$, and cannot be neglected. It contributes the following orbital matrix element for pion decay of the $D_{1}$ meson:

$$
\begin{aligned}
\mathcal{M}_{2 \mathrm{~S}}^{\mathrm{OGE}} \simeq & \frac{1}{\pi} \int_{0}^{\infty} d r^{\prime} r^{\prime} \int_{0}^{\infty} d r r V_{g}\left(\sqrt{\frac{r^{2}+r^{\prime 2}}{2}}\right) \int_{0}^{\infty} d P P^{2} \int_{-1}^{1} d z f_{\mathrm{BS}}(P, z) \\
& \frac{4 m^{2}}{\sqrt{4 E E^{\prime}(E+m)\left(E^{\prime}+m\right)}} \frac{2 M+E_{c}^{\prime}+E_{c}}{\sqrt{4 E_{c}^{\prime} E_{c}\left(E_{c}^{\prime}+M\right)\left(E_{c}+M\right)}} \\
& {\left[u_{0}^{\prime}\left(r^{\prime}\right) u_{1}(r)-u_{0}\left(r^{\prime}\right) u_{1}^{\prime}(r)-2 \frac{u_{0}\left(r^{\prime}\right) u_{1}(r)}{r}\right] }
\end{aligned}
$$




$$
j_{0}\left(r^{\prime} \sqrt{P^{2}+\frac{k^{2}}{16}+\frac{P k z}{2}}\right) j_{0}\left(r \sqrt{P^{2}+\frac{k^{2}}{16}-\frac{P k z}{2}}\right) .
$$

The effective one-gluon exchange potential $V_{g}(r)$ that is appropriate for the $D$ meson systems and which takes into account the screened running color coupling $\alpha_{s}$ has been calculated in ref. [6]. This potential function may be well parametrized as $V_{g}(r)=-A \arctan (B r) / r$, with $\mathrm{A}=1.1899$ and $\mathrm{B}=3.39768 / \mathrm{fm}$. Using this form, the numerical value $\mathcal{M}_{2 \mathrm{~S}}^{\mathrm{OGE}}=132 \mathrm{MeV}$ is obtained. In the static non-relativistic limit, the one-gluon exchange contribution reduces to

$$
\mathcal{M}_{2 \mathrm{~S}}^{\mathrm{OGE}} \simeq \frac{1}{M} \int_{0}^{\infty} d r V_{g}(r)\left[u_{0}^{\prime}(r) u_{1}(r)-u_{0}(r) u_{1}^{\prime}(r)-2 \frac{u_{0}(r) u_{1}(r)}{r}\right] j_{0}\left(\frac{k r}{2}\right) .
$$

The two-quark mechanism shown in Fig. 3 contributes to the $D$-wave pion decay amplitude from the axial charge operator as well. As in the case of $S$-wave pion decay, this effect may be taken into account by adding to the one-body matrix element $\mathcal{M}_{1 \mathrm{D}}$ the matrix element that is associated with the exchange charge contribution. For the confining interaction, this matrix element may be obtained similarly to eq. (41), giving

$$
\begin{aligned}
\mathcal{M}_{2 \mathrm{D}}^{\mathrm{conf}} \simeq & \frac{1}{\pi} \int_{0}^{\infty} d r^{\prime} r^{\prime} \int_{0}^{\infty} d r r V_{c}\left(\sqrt{\frac{r^{2}+r^{\prime 2}}{2}}\right) \int_{0}^{\infty} d P P^{2} \int_{-1}^{1} d z f_{\mathrm{BS}}(P, z) \\
& \left(\frac{3}{2} \frac{P^{2}\left(1-z^{2}\right)}{P^{2}+\frac{k^{2}}{16}-\frac{P k z}{2}}-1\right)\left[u_{0}^{\prime}\left(r^{\prime}\right) u_{1}(r)-u_{0}\left(r^{\prime}\right) u_{1}^{\prime}(r)+\frac{u_{0}\left(r^{\prime}\right) u_{1}(r)}{r}\right] \\
& \frac{4 m}{\sqrt{4 E E^{\prime}(E+m)\left(E^{\prime}+m\right)}} \sqrt{\frac{E_{c}^{\prime}+M}{2 E_{c}^{\prime}}} \sqrt{\frac{E_{c}+M}{2 E_{c}}}\left(1-\frac{P^{2}-k^{2} / 4}{\left(E_{c}^{\prime}+M\right)\left(E_{c}+M\right)}\right) \\
& j_{0}\left(r^{\prime} \sqrt{P^{2}+\frac{k^{2}}{16}+\frac{P k z}{2}}\right) j_{2}\left(r \sqrt{P^{2}+\frac{k^{2}}{16}-\frac{P k z}{2}}\right) .
\end{aligned}
$$

A similar expression may readily be constructed for the $D$-wave contribution from the one-gluon exchange interaction by the techniques outlined in this section. The numerical value of the matrix element (45) is of the same magnitude $(1 \mathrm{MeV})$ as that of eq. (36) and thus completely insignificant. As the one-gluon exchange contribution is smaller by a factor $m / M$, it will not be considered in this paper. In the nonrelativistic limit, the expression (45) reduces to

$$
\mathcal{M}_{2 \mathrm{D}}^{\operatorname{conf}} \simeq-\frac{1}{m} \int_{0}^{\infty} d r V_{c}(r)\left[u_{0}^{\prime}(r) u_{1}(r)-u_{0}(r) u_{1}^{\prime}(r)+\frac{u_{0}(r) u_{1}(r)}{r}\right] j_{2}\left(\frac{k r}{2}\right) .
$$

The numerical values of the matrix elements for $S$-wave pion decay of the $D_{1}$ meson are given in Table 4 . The matrix element of the two-quark contribution $\mathcal{M}_{2 \mathrm{~S}}^{\text {conf }}$, eq. (41), is of the same magnitude as the single quark contribution, eq. (31), and has opposite sign. The corresponding matrix element from the one-gluon exchange interaction, eq. (43), represents a somewhat smaller contribution. Addition of these three matrix elements reduces the net matrix element for $S$-wave pion decay of the $D_{1}$ state by about a factor 3 as shown in Table 4 . With this smaller value the net contribution of $S$-wave pion decay to $\left(D_{1} \rightarrow D^{*} \pi\right)$ becomes quite small and comparable to that from the axial current operator, as 
indicated in Table 5. It should, however, be noted that the results are very sensitive to the exact form and composition of the interaction Hamiltonian used in the calculations.

In the case of the $D_{2}^{*}$ mesons the calculated width falls below the current empirical range by about $40 \%$ if $g_{A}^{q}=0.87$. With $g_{A}^{q}=1$ the calculated values are only slightly below the empirical values. These results leave room for about $5-10 \mathrm{MeV}$ for the contribution from $\pi \pi$ decay. This is what would be expected on the basis of analogy with the decay pattern of the strange $K_{2}^{*}(1430)$ meson, which should have a structure similar to that of the $D_{2}^{*}(2460)$, once the charm quark is replaced by a strange quark. If the nonrelativistic values for the orbital matrix elements $\mathcal{M}_{1}$ given in Table 3 are used in the calculation, the calculated width for the $D_{2}^{*}$ meson would be well above the empirical range. The ratio of the calculated pion decay widths of the $D_{2}^{*}$ meson and the $D_{1}$ meson is 1.2. This result is compatible with the current empirical value $\sim 1.3$.

\begin{tabular}{c|c|c|c|c} 
Decay & $\mathcal{M}_{1 \mathrm{~S}}$ & $\mathcal{M}_{2 \mathrm{~S}}^{\text {conf }}$ & $\mathcal{M}_{2 \mathrm{~S}}^{\text {OGE }}$ & Total \\
\hline \hline$D_{1} \rightarrow D^{*} \pi, \mathrm{NR}$ & -1194 & +450 & +411 & -333 \\
& & & & \\
$D_{1} \rightarrow D^{*} \pi, \mathrm{REL}$ & -592 & +278 & +133 & -181
\end{tabular}

Table 4: Matrix elements in $\mathrm{MeV}$ of the single quark and two-quark operators for the $S$-wave axial charge contribution to the decay $D_{1} \rightarrow D \pi$. The resulting net contribution to this decay mode is also given. The labels NR and REL indicate that the non-relativistic and relativistic expressions have been used respectively.

\begin{tabular}{l|c|c|c|c|c}
\multicolumn{1}{c|}{ Decay } & Current & Charge & Total & $g_{A}^{q}=1$ & Experiment \\
\hline \hline$D_{1} \rightarrow D^{*} \pi$ & $4.2(3.4) \mathrm{MeV}$ & $6.1 \mathrm{MeV}$ & $10.3 \mathrm{MeV}$ & $13.6 \mathrm{MeV}$ & $18.9_{-3.5}^{+4.6} \mathrm{MeV}$ \\
& $8.1(6.7) \mathrm{MeV}$ & - & $8.1 \mathrm{MeV}$ & $10.6 \mathrm{MeV}$ & $?$ \\
$D_{2}^{*} \rightarrow D \pi$ & & & & & \\
& & & & & \\
$D_{2}^{*} \rightarrow D^{*} \pi$ & $3.9(3.1) \mathrm{MeV}$ & - & $\mathrm{MeV}$ & $5.1 \mathrm{MeV}$ & $?$ \\
$D_{2}^{*} \rightarrow D \pi+D^{*} \pi$ & $11.9(9.9) \mathrm{MeV}$ & - & $11.9 \mathrm{MeV}$ & $15.7 \mathrm{MeV}$ & $25_{-7}^{+8} \mathrm{MeV}$
\end{tabular}

Table 5: Calculated and empirical pion decay widths of the $D_{1}$ and $D_{2}^{*}$ mesons driven by the axial current and charge operators respectively, for $g_{A}^{q}=0.87$. The empirical values are total widths [12], which should mainly be due to pion decay to the ground state. The numbers in parentheses are the decay widths obtained without the axial exchange current contribution. The calculated values are also shown for $g_{A}^{q}=1$.

In the case of the $D_{1}$ mesons the total width also obtains a significant contribution from $S$-wave pion decay. Here it has been assumed that the empirical widths are mainly due to pion decay to the ground state $D$ and $D^{*}$ mesons. In addition, even though the present empirical data on the $L=1$ $D$ mesons is severely limited, the ratio of $D \pi$ to $D^{*} \pi$ decay of the $D_{2}^{*}$ meson has been measured [12]. From the results in Table 5, we obtain 


$$
\frac{\Gamma\left(D_{2}^{*} \rightarrow D \pi\right)}{\Gamma\left(D_{2}^{*} \rightarrow D^{*} \pi\right)}=2.1
$$

which is in agreement with the experimentally determined value $2.3 \pm 0.6$ for the neutral $D_{2}^{*}$ meson 12 ] (The measured value for the charged $D_{2}^{*}$ meson has much larger statistical uncertainties). Note that in Table 5 , the quoted experimental values are for the neutral $D_{1}$ and the charged $D_{2}^{*}$ mesons. The corresponding value for the neutral $D_{2}^{*}$ meson is $23 \pm 5 \mathrm{MeV}$ [12]. The available data for the charged $D_{1}^{*}$ meson is considerably poorer, since it has only recently been discovered.

\section{Pion decay widths of the $D$ mesons with $L=1, J=0,1$}

Two $D$ meson resonances with $L=1$ remain to be found experimentally. In the $L S$ basis these are the spin singlet state $D_{1}^{*}$ with $J=1$ and the spin triplet state $D_{0}^{*}$ with $J=0$. In this context, it is worth noting that although the 'star' in the labeling notation $D^{*}$ is usually reserved for states with $J^{P}=0^{+}, 1^{-}, 2^{+}, \cdots$, it has become conventional to label the spin singlet state $D_{1}^{*}$ to distinguish it from the spin triplet state $D_{1}$. In the Heavy Quark Symmetry (HQS) framework, the $D_{1}^{*}$ and $D_{0}^{*}$ mesons correspond to states with light quark angular momentum $j_{q}=1 / 2$. Both of these states may pion decay by $D$-wave decay through the axial current operator as well as by $S$-wave decay through the axial charge operator. Consider first the (mainly) singlet $D_{1}^{*}$ state. Because of the spin dependence of the pion-quark coupling, it follows that this state can only pion decay to the triplet $D^{*}$ meson. To derive the expression for the width of this decay mode, the following additional spin sums are required:

$$
\begin{aligned}
S_{s} & =\frac{1}{3} \sum_{M=-1}^{1}\left\langle 10,1 M\left|\vec{\sigma}_{q} \cdot \vec{k}\right| 00,00\right\rangle\left\langle 00,00\left|\vec{\sigma}_{q} \cdot \vec{k}\right| 10,1 M\right\rangle \\
& =\frac{1}{3} \sum_{M=-1}^{1}\left\langle 10,1 M\left|-\frac{1}{6} S_{12}(\vec{k})\right| 10,1 M\right\rangle
\end{aligned}
$$

for spin singlet final states, and

$$
\begin{aligned}
S_{t} & =\frac{1}{3} \sum_{M=-1}^{1} \sum_{m=-1}^{1}\left\langle 10,1 M\left|\vec{\sigma}_{q} \cdot \vec{k}\right| 01,1 m\right\rangle\left\langle 01,1 m\left|\vec{\sigma}_{q} \cdot \vec{k}\right| 10,1 M\right\rangle \\
& =\frac{1}{3} \sum_{M=-1}^{1}\left\langle 10,1 M\left|k^{2}+\frac{1}{6} S_{12}(\vec{k})\right| 10,1 M\right\rangle
\end{aligned}
$$

for spin triplet final states. From these expressions, it follows that the decay mode $D_{1}^{*} \rightarrow D \pi$ is forbidden, since the tensor operator $S_{12}$ has a vanishing matrix element between spin singlet states. The resulting expressions for the pionic decay width of the $D_{1}^{*}$ state driven by the axial current operator is then

$$
\Gamma_{A}\left(D_{1}^{*} \rightarrow D^{*} \pi\right)=\frac{3}{8 \pi} \frac{E_{D^{*}}}{M_{D_{1}^{*}}}\left(\frac{g_{A}^{q}}{f_{\pi}}\right)^{2} k^{3} \mathcal{M}_{1}^{2} .
$$


The corresponding expression for the pion decay width of the spin triplet $D_{0}^{*}$ state may, using the spin sums given in eqs. (17) and (18), be expressed as

$$
\Gamma_{A}\left(D_{0}^{*} \rightarrow D \pi\right)=\frac{3}{8 \pi} \frac{E_{D}}{M_{D_{0}^{*}}}\left(\frac{g_{A}^{q}}{f_{\pi}}\right)^{2} k^{3} \mathcal{M}_{1}^{2} .
$$

The axial charge operator also contributes to these decay modes. The expressions for these $S$-wave contributions may be obtained as

$$
\Gamma_{C}\left(D_{1}^{*} \rightarrow D^{*} \pi\right)=\frac{3}{64 \pi} \frac{E_{D^{*}}}{M_{D_{1}^{*}}}\left(\frac{g_{A}^{q}}{f_{\pi}}\right)^{2}\left(\frac{\omega_{\pi}}{m}\right)^{2} k \mathcal{M}_{1 S}^{2}
$$

for the $D_{1}^{*}$ meson, and

$$
\Gamma_{C}\left(D_{0}^{*} \rightarrow D \pi\right)=\frac{3}{64 \pi} \frac{E_{D}}{M_{D_{0}^{*}}}\left(\frac{g_{A}^{q}}{f_{\pi}}\right)^{2}\left(\frac{\omega_{\pi}}{m}\right)^{2} k \mathcal{M}_{1 S}^{2}
$$

for the $D_{0}^{*}$ meson. The matrix elements required for the evaluation of these decay widths are given in Table 6, and the pion momenta and masses used can be found in Table 11. The resulting calculated pion decay widths of the $D_{1}^{*}$ and $D_{0}^{*}$ mesons are given in Table 7 . It is evident that the $S$-wave contributions are dominant in these cases, although the $D$-wave decays also contribute significantly. In the HQS framework, only $S$-wave pion decay is allowed to contribute [8].

\begin{tabular}{c|c|c|c|c|c|c} 
Decay & $\mathcal{M}_{1}$ & $\mathcal{M}_{1}^{\text {ex }}$ & $\mathcal{M}_{1 \mathrm{~S}}$ & $\mathcal{M}_{1 \mathrm{~S}}^{\text {conf }}$ & $\mathcal{M}_{1 \mathrm{~S}}^{\mathrm{OGE}}$ & $\mathcal{M}_{1 \mathrm{~S}}^{\mathrm{TOT}}$ \\
\hline \hline$D_{1}^{*} \rightarrow D^{*} \pi$ & 0.086 & 0.010 & $-598 \mathrm{MeV}$ & $+284 \mathrm{MeV}$ & $+135 \mathrm{MeV}$ & $-179 \mathrm{MeV}$ \\
& & & & & & \\
$D_{0}^{*} \rightarrow D \pi$ & 0.106 & 0.011 & $-578 \mathrm{MeV}$ & $+267 \mathrm{MeV}$ & $+130 \mathrm{MeV}$ & $-181 \mathrm{MeV}$
\end{tabular}

Table 6: Matrix elements of the axial current and charge operators for the decays of the $D_{1}^{*}$ and $D_{0}^{*}$ mesons. The matrix elements $\mathcal{M}_{1 \mathrm{~S}}^{\mathrm{TOT}}$ represent the resulting net contribution to $S$-wave pion decay from the axial charge operator, eq. (28), when the two-body matrix elements from Section 3.4 are added to the single quark contribution.

\begin{tabular}{c|c|c|c|c} 
Decay & Current & Charge & Total & $g_{A}^{q}=1$ \\
\hline \hline$D_{1}^{*} \rightarrow D^{*} \pi$ & $2.8(2.3) \mathrm{MeV}$ & $7.2 \mathrm{MeV}$ & $10.0 \mathrm{MeV}$ & $13.2 \mathrm{MeV}$ \\
& & & & \\
$D_{0}^{*} \rightarrow D \pi$ & $7.9(6.5) \mathrm{MeV}$ & $13.0 \mathrm{MeV}$ & $20.9 \mathrm{MeV}$ & $27.7 \mathrm{MeV}$
\end{tabular}

Table 7: Predicted pion decay widths of the $D_{1}^{*}$ and $D_{0}^{*}$ mesons driven by the axial current and charge operators respectively, for $g_{A}^{q}=0.87$. The empirical values are total widths 12, which should mainly be due to pion decay to the ground state. The numbers in parentheses are the decay widths obtained without the axial exchange current contribution. The calculated values are also shown for $g_{A}^{q}=1$. 
The pion widths of the $D_{1}^{*}$ and $D_{0}^{*}$ states have been predicted to be much larger in the previous literature [8, 20], although in ref. [8] a considerable reduction of these widths was already hinted at. One reason for the smallness of the values obtained here is the more complete degree of suppression of $S$-wave pion decay modes achieved here. This suppression was already shown to be necessary in order to avoid a large overprediction of the width of the $D_{1}$ meson. Since eqs. (30) and (52) suggest that

$$
\Gamma_{C}\left(D_{1}^{*} \rightarrow D^{*} \pi\right)=\frac{3}{2} \Gamma_{C}\left(D_{1} \rightarrow D^{*} \pi\right),
$$

if the pion momenta $k$ are the same, it immediately follows that the $S$-wave widths of these two states are of the same order of magnitude. Consequently, as the triplet $D_{1}$ state does not have any large $S$-wave contribution to its pionic width, it follows that the singlet $D_{1}^{*}$ state should be rather narrow as well. The calculated pion decay widths of the $D_{1}^{*}$ and $D_{0}^{*}$ mesons are here further reduced by the significant spin-orbit splittings in the $P$-shell, see Fig. 1. The spectrum obtained in ref. [6] predicts the traditional "hydrogen-like" ordering of the $P$-wave states, which is supported by recent NRQCD lattice studies [9]. Since the decay rates are very sensitive to the amount of phase space available, this effect turns out to be quite significant. Consequently, the $D_{2}^{*}$ meson has considerably more phase space available than the other $P$-shell states.

In this context, it is instructive to note that the pion decay rates of the $K_{0}^{*}(1430)$ and $K_{2}^{*}(1430)$, which are analogous to the $D_{0}^{*}$ and $D_{2}^{*}$ states, have been experimentally determined [12]. The ratio of the widths of the $K \pi$ decays of these strange mesons is particularly interesting, since the pion momenta in these decays are exactly equal. The ratio of the width of $K_{0}^{*} \rightarrow K \pi$ to that of $K_{2}^{*} \rightarrow K \pi$ is $\sim 5.7$. Using eqs. (51) and (53) with the pion momentum and matrix elements of the $D_{2}^{*}$ meson, the corresponding ratio for the $D$ mesons comes to $\sim 5.4$, which supports the conclusion that the pionic width of the $D_{0}^{*}$ state should not be much larger than $\sim 30 \mathrm{MeV}$, as indicated in Table 7 .

It should however be noted that as a consequence of the cancellation between the exchange charge operator contribution and the contribution from the single quark axial charge operator, the $S$-wave decay widths are very sensitive to the strength, form and composition of the interaction Hamiltonian of the $q \bar{Q}$ system. Empirical determination of the decay widths of the $D_{0}^{*}$ and $D_{1}^{*}$ mesons should as a consequence be able to provide useful information on the forms of these interactions.

\section{Discussion}

The present calculation of the pion decay widths of the excited charm mesons indicates that the chiral quark model description is compatible with the extant experimental data. The underprediction of the total decay widths of the $L=1$ charm mesons is to be expected, as the pion decay pattern of the strange meson $L=1$ states as e.g. the $K_{2}^{*}(1430)$, suggests that $\pi \pi$ decay should give a contribution to the decay width of the $D_{2}^{*}$ meson that is about a third as large as that of single pion decay.

This point illustrates the difference between the present relativistic Hamiltonian method and the heavy quark effective field theory (HQET) method, in which at the present stage the effective coupling strength of pions to light constituent quarks has to be determined by the empirical width of one of the orbitally excited $D$ mesons under the assumption that the width is solely due to single pion decay [20]. Given that restriction, the present results for the decay widths of the $D_{1}$ and $D_{2}^{*}$ mesons are overall rather similar to those in ref. [20], although some details as e.g. the ratio between the decay widths to 
$D \pi$ and $D^{*} \pi$ of the $D_{2}^{*}$, eq. (47), obtained there is 3.5 and thus somewhat above the empirical value $2.3 \pm 0.6$.

The present calculation, which was carried out within the framework of the Blankenbecler-Sugar reduction of the Bethe-Salpeter equation, affirms the observation of ref. 8], that a relativistic treatment of the $q \bar{Q}$ system is required, as a realistic description of the pionic decay widths is not attainable with a non-relativistic description. The framework of the Blankenbecler-Sugar equation brings the advantage of formal similarity to the conventional non-relativistic quantum mechanical treatment. In this approach the exchange currents that are associated with the scalar confining and vector onegluon exchange interactions play an important role in suppressing the otherwise unrealistically large amplitude for $S$-wave pion decay of the $D_{1}$ meson. This also has the effect of drastically reducing the calculated widths of the singlet $D_{1}^{*}$ and triplet $D_{0}^{*}$ states, which are predicted to be extremely broad in the HQS framework. In that framework, the allowed pion decay modes are determined by the total angular momentum of the light constituent quark, which is appropriate if the spins of the light and heavy quarks are decoupled. This is the case if the heavy quark mass is very large compared to that of the light constituent quark. However, the empirical $D$ meson spectrum shown in Fig. 1 indicates that the spins of the quarks are strongly coupled, as the $D^{*}-D$ splitting is of the order $\sim 130 \mathrm{MeV}$. In view of this, the present treatment is more general since it is not restricted to the case when the heavy quark mass is infinite. The present work incorporates all decay modes that contribute in that limit [8]. An analogous treatment within the framework of HQS requires an $1 / M_{Q}$ expansion along with the introduction of new parameters, which have to be fitted to experimental data.

The main goal of studying the pion decays of the charm mesons is that of determining the numerical value of the axial coupling $g_{A}^{q}$ of the light constituent quarks. As long as the absolute values of the widths of the $D^{*}$ mesons are not known, this goal cannot be conclusively attained. Nevertheless both the present study, as well as the earlier investigation in ref. [8], suggests that the standard values of $g_{A}^{q}$, being somewhat below 1 appear to apply well in the case of the pion decays of the charm mesons.

\section{Acknowledgments}

K.O.E.H. and C.J.N. thank the Finnish Society of Science and Letters for a stipend. Research supported in part by the Academy of Finland under contracts 43982 and 44903. 


\section{Appendix A}

Because of the smooth radial dependence of the scalar confining and the one-gluon exchange interactions in the $q \bar{Q}$ system, the reduced wave functions of the low-lying states of the $q \bar{Q}$ system may be well approximated by the following simple expression:

$$
u_{l}(r) \simeq N_{l} r^{l} e^{-d r^{3 / 2}}
$$

Here $m_{r}$ is the reduced mass of the $q \bar{Q}$ system and $c$ is the confining string tension. The coefficient $d$ is defined as

$$
d=\frac{2}{3} \sqrt{2 m_{r} c}
$$

and $N_{l}$ is a normalization factor chosen so that

$$
\int_{0}^{\infty} d r u_{l}(r)^{2}=1
$$

These approximate expressions have the correct behavior at both small and large values of the quark separation $r$. For $l=0,1$ the expressions for the normalization constants may, with the aid of eq. (56), be obtained as

$$
\begin{aligned}
& N_{0}=\frac{4}{3} \sqrt{3 m_{r} c} \\
& N_{1}=\frac{2^{11 / 3}}{3^{7 / 3}} \frac{\left(m_{r} c\right)^{5 / 3}}{\sqrt{\Gamma(10 / 3)}} .
\end{aligned}
$$

The approximate reduced wave functions $u_{0}(r)$ and $u_{1}(r)$ are compared to the corresponding numerical solutions to the Blankenbecler-Sugar equation in Fig. 2. 


\section{References}

[1] F. Gross, Phys. Rev. 186 (1969) 1448

[2] R. Blankenbecler and R. Sugar, Phys. Rev. 142 (1966) 1051

[3] A.A. Logunov and A.N. Tavkhelidze, Nuovo Cimento 29 (1963) 380

[4] J. Zeng, J.W. Van Orden and W. Roberts, Phys. Rev. D52 (1995) 5229, hep-ph/9412269

[5] D. Ebert, V.O. Galkin and R. Faustov, Phys. Rev. D57 (1998) 5663, erratum ibid. D59 (1999) 1019902, hep-ph/9712318

[6] T.A. Lähde, C.J. Nyfält and D.O. Riska, Nucl. Phys. A674 (2000) 141 hep-ph/9908485

[7] T.A. Lähde, C.J. Nyfält and D.O. Riska, Nucl. Phys. A645 (1999) 587, hep-ph/9808438

[8] J.L. Goity and W. Roberts, Phys. Rev. D60 (1999) 034001, hep-ph/9809312

[9] R. Lewis and R.M. Woloshyn, hep-lat/0003011

[10] N. Isgur and M. Wise, Phys. Rev. Lett. 66 (1991) 1130

[11] A.F. Falk and T. Mehen, Phys. Rev. D53 (1996) 321, hep-ph/9507311

[12] D.E. Groom et al., Eur. Phys. Journal C15, 1 (2000)

[13] S. Weinberg, Phys. Rev. Lett. 65 (1990) 1181

[14] S. Weinberg, Phys. Rev. Lett. 67 (1991) 3473

[15] D.A. Dicus et al., Phys. Lett. B284 (1992) 384

[16] K. Dannbom et al., Nucl. Phys. A616 (1997) 555, hep-ph/9610384

[17] K. Tsushima and D.O. Riska, Nucl. Phys. A549 (1992) 313

[18] A.C. Mattingly and P.M. Stevenson, Phys. Rev. D49 (1994) 437, hep-ph/9307266

[19] M. Kirchbach, D.O. Riska and K. Tsushima, Nucl. Phys. A542 (1992) 616

[20] A. Deandrea et al., JHEP 9902, 021 (1999), hep-ph/9901266 